\title{
Breathomics in Major Depressive Disorder
}

\author{
$\underline{\text { T. Frodl }}^{1}$, M. Lüno ${ }^{1}$, G. Meyer-Lotz ${ }^{1}$, L. Gbaoui², C. Metzger ${ }^{1}$, D. Gescher ${ }^{1}$, J. Steiner ${ }^{1}$, C. Hoeschen ${ }^{2}$ \\ ${ }^{1}$ Otto von Guericke University, Magdeburg, Germany, \\ 1. Medical Faculty, Psychiatry and Psychotherapy, 2. Medical Systems
} Goal of the study is to investigate whether expiratory breath gas includes volatile organic compounds (VOCs) that
can distinguish patients with clinical diagnosed MDD from age and gender matched healthy controls and whether
these signatures are associated with severity of depression and anxiety.

\section{Background:}

Brain disorders cost Europe almost $€ 800$ billion (US $\$ 1$ trillion) a year - more than cancer, cardiovascular disease and diabetes put together. In particular, for psychiatric disorders no in-vivo and easy to apply biomarkers are available that could help in diagnosis or therapy.

E.g. for major depressive disorder (MDD), there is compelling evidence that environmental stress, in particular through the action of glucocorticoids, induces enhancement of excitatory (glutamatergic) neurotransmission leading to dendritic remodeling in a number of brain regions associated with behavioural changes. This hypothesis is known as glutamate hypothesis of MDD. Brain's glutamate cannot easily be measured, but indirectly it might be possible to obtain signatures associated with the glutamatergic and the stress system.

Because the lungs act as a gas exchanger between the internal and external environment, the impact of disorders like MDD may be easily assessed through the analysis of exhaled breath. Breath gas analysis was recently successfully applied by our cooperation partner Prof. Hoeschen at the department for medical techniques of our university in humans and animal experimental research. This technique was recently successfully applied in a human study with patients with diabetes, thus demonstrating clinical applicability (personal communication and (1)).

Breath gas analysis is fully installed in our department of psychiatry and we are able to obtain 100 signatures from the breath gas.

\section{Results and Discussion:}

70 molecular weight masses gave signal in most of our participants. Interestingly, molecular weight 68 showed a significant time by diagnosis interaction (see Figure $1 \mathrm{~A}-\mathrm{C}$ ). Posthoc analysis showed that in healthy controls this VOC decreased gradually from awakening over 1 hour, whereas this was not the case in patients with MDD. Patients with MDD had significantly lower concentration of the VOC, in particular after awakening and still 30 minutes later. Similar, concentration of molecular weights 39 and 40 showed significant time by diagnosis interactions and concentration of weight 39 was significantly lower in patients with MDD at baseline.

The first results are promising and it will be important to see how these results develop in a larger sample. In a next step we also aim to identify the chemical substances of the VOCs belonging to these signatures.

\section{Materials and Methods:}

Patients with major depressive disorder (MDD) according to DSM-V have been included as well as age and gender matched healthy controls (age 18-65). The recruitment is currently ongoing. Diagnosis was confirmed using the SCID-V I and II diagnostic tools in addition to the clinical diagnosis made by 2 consultants. Exclusion criteria are any other psychiatric disorder and internal or neurological diseases affecting the central nervous system. Healthy controls are from the general population.

Table: Description of current study population, BDI: Beck Depression Inventory-II, HDRS: Hamilton Depression Rating Scale

\begin{tabular}{|l|l|l|l|}
\hline & $\begin{array}{l}\text { HC } \\
\mathbf{N}=5\end{array}$ & $\begin{array}{l}\text { MDD } \\
\mathbf{N}=13\end{array}$ & Difference, $\mathrm{p}$-value \\
\hline Age & $30.6 \pm 10.7$ & $40.2 \pm 14.7$ & $\mathrm{t}=-1.3, \mathrm{p}=0.21$ \\
\hline Gender $(\mathrm{m} / \mathrm{f})$ & $4 / 1$ & $5 / 8$ & $\mathrm{Chi}=2.5, \mathrm{p}=0.11$ \\
\hline Height & $179.8 \pm 5.4$ & $169.9 \pm 12.9$ & $\mathrm{t}=1.6, \mathrm{p}=0.13$ \\
\hline Weight & $76.0 \pm 10.7$ & $76.7 \pm 19.2$ & $\mathrm{t}=-0.08, \mathrm{p}=0.94$ \\
\hline BDI & $0.8 \pm 1.8$ & $34.1 \pm 10.1$ & $\mathrm{t}=11.0, \mathrm{p}<0.001$ \\
\hline HDRS & $0 \pm 0$ & $16.5 \pm 3.6$ & $\mathrm{t}=16.6, \mathrm{p}<0.001$ \\
\hline
\end{tabular}

Psychopathology has been documented after clinical interviews using the following scales: Hamilton Depression Scale (HDRS) and Clinical Global Impression (CGI). Patients filled in the selfrater questionnaires Beck Depression Inventory (BDI), Beck Anxiety Inventory (BAI), and the Pittsburgh Sleep Quality Index (PSQI).
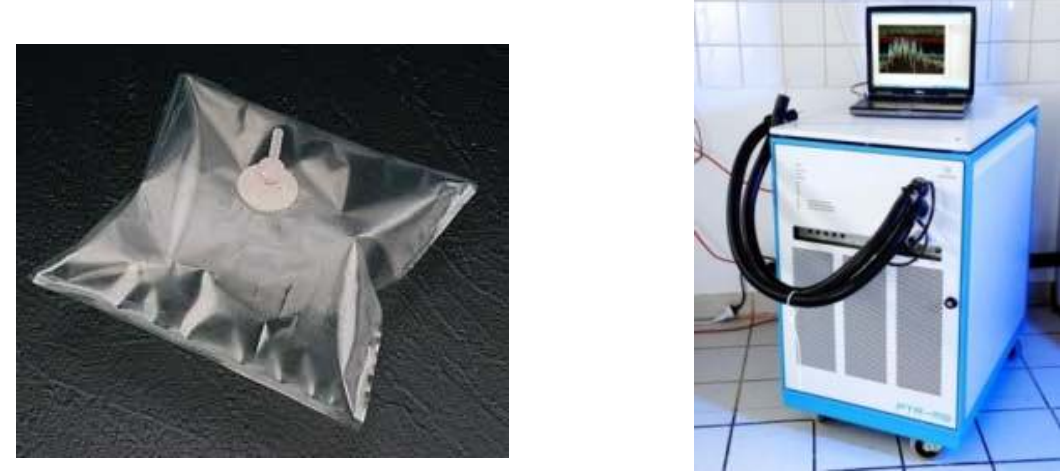

Breath gas Procedure and biological samples: Breath gas was analyzed by use of proton-transfer-reaction-mass spectrometer (PTR-MS) (2) (3). It has been shown that stress hormones and inflammatory markers underlie a certain day rhythm in depression and lead - depending on the severity of depression- to an overwhelming or lack of stress hormones in comparison to healthy controls (U-shape association) (4). Thus, breath gas was measured at awakening, as well as 30 and 60 minutes later.

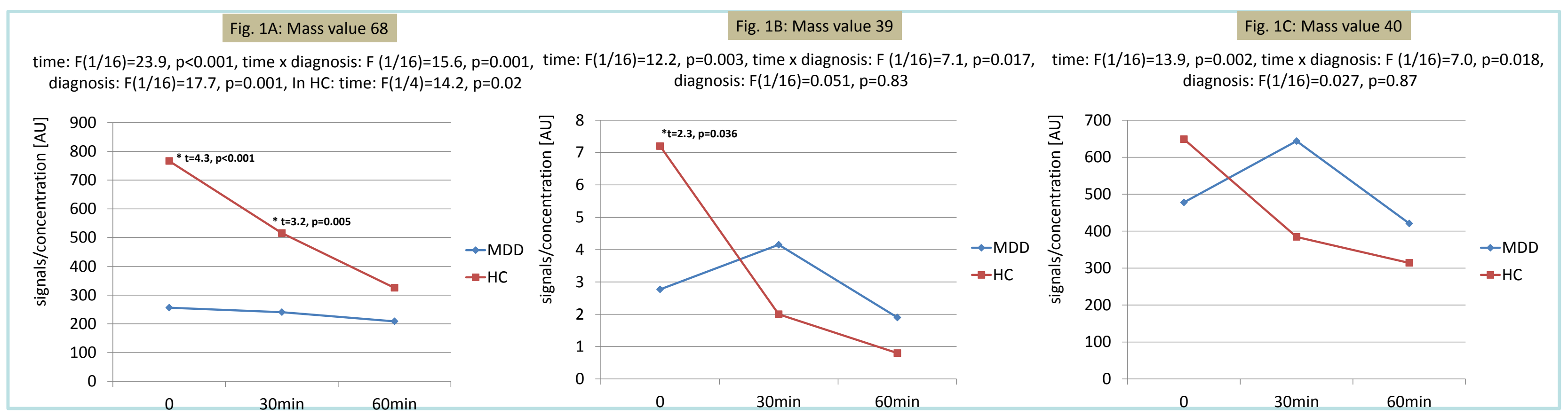

1. Siegel AP, Daneshkhah A, Hardin DS, Shrestha S, Varahramyan K, Agarwal M. Analyzing breath samples of hypoglycemic events in type 1 diabetes patients: towards developing an alternative to diabetes alert dogs. J Breath Res. 2017 ; 11(2):026007. 2. Hunt JF, Erwin E, Palmer L, Vaughan J, Malhotra N, Platts-Mills TA, et al. Expression and activity of pH-regulatory glutaminase in the human airway epithelium. Am J Respir Crit Care Med. 2002;165(1):101-7. 